\title{
EHMTI-0266. Validation of a sham manipulative procedure: a new protocol for conducting placebo-control clinical trials in manual therapy
}

\author{
A Chaibi ${ }^{1 *}$, J Saltyte Benth², P Tuchin ${ }^{3}$, MB Russell ${ }^{4}$ \\ From 4th European Headache and Migraine Trust International Congress: EHMTIC 2014 \\ Copenhagen, Denmark. 18-21 September 2014
}

\section{Background}

Few manual therapy studies have attempted to conduct placebo-control clinical trials. Thus, quantification of alleged placebo effects consequently becomes difficult.

\section{Aim}

To investigate and validate a new placebo intervention for spinal manipulative therapy clinical trials, i.e. sham manipulation, and investigate the feasibility of a short deblinding questionnaire.

\section{Method}

A single blinded, prospective randomized, placebocontrolled trial with 1 month baseline and 3 months treatment with 12 treatments. 104 participants diagnosed with migraine were equally randomized into 1 of 3 groups: (i) chiropractic spinal manipulative therapy (CSMT), (ii) placebo (sham manipulation), (iii) control group (continued usual management). The participants filled in questionnaire on de-blinding after each treatment session. Primary end-point was the rate of successful blinding through de-blinding questionnaires given after each treatment session.

\section{Results}

772 out of 840 individual cases were analyzed, only $8.1 \%$ out of all cases missed their appointment. The unadjusted result shows that both the active and placebo group believed they received active treatment with odds ratio (OR) of $\geq 88.9$ and $\geq 80.0$ respectively. Due to strong cluster effect in our data, logistic regression model was used to adjust for repeated measures which showed a significant OR of $>10$ in both the active and the placebo group.

\section{Conclusion}

This is the first study to successfully demonstrate a manipulative sham procedure over a full length treatment period, assessing the placebo group with deblinding questionnaire after each session which thus, could be incorporated in future clinical trials.

No conflict of interest.

\section{Authors' details}

${ }^{1}$ Head and Neck Research Group, University of Oslo and Reserach Centre Akershus University Hospital, Lorenskog, Norway. ${ }^{2} \mathrm{H} \varnothing \mathrm{KH}$ Research Centre, Institute of Clinical Medicine Akershus University Hospital, Lorenskog, Norway. ${ }^{3}$ Department of Chiropractic, Macquarie University, Sydney, Australia. ${ }^{4}$ Head and Neck Research Group, Research Centre Akershus University Hospital, Lorenskog, Norway.

Published: 18 September 2014

doi:10.1186/1129-2377-15-S1-E7

Cite this article as: Chaibi et al:: EHMTI-0266. Validation of a sham manipulative procedure: a new protocol for conducting placebo-control clinical trials in manual therapy. The Journal of Headache and Pain 2014 15(Suppl 1):E7. 